\title{
Nature of the Glomerular Capillary Injury in Human Membranous Glomerulopathy
}

\author{
O. Shemesh, J. C. Ross, W. M. Deen, G. W. Grant, and B. D. Myers \\ Departments of Medicine and Pathology, Stanford University School of Medicine, Stanford, California 94305; Department of Chemical \\ Engineering, Massachusetts Institute of Technology, Cambridge, Massachusetts 02139
}

\begin{abstract}
A differential solute clearance technique was used to evaluate glomerular capillary wall function in 20 patients with membranous glomerulopathy and massive proteinuria. The clearance of inulin, the filtration fraction, and the fractional clearance of uncharged dextrans of a radius of 28-48 $\AA$ were depressed significantly below control values in 20 healthy volunteers $(P<0.01)$. In contrast, the fractional clearance of dextrans of radius $>\mathbf{5 0}$ $\AA$ was elevated markedly. A theoretical model of solute transport that depicts the major portion of the glomerular capillary wall as an isoporous membrane and the minor portion as a nondiscriminatory shunt pathway revealed the calculated gomerular ultrafiltration coefficient to be five times lower and mean pore radius of the major membrane component to be $4 \AA$ smaller than control values. However, the fraction of filtrate volume permeating the shunt pathway was three- to fourfold above control values and correlated strongly in individual patients with the fractional clearance of albumin $(r=0.76)$ and of $\operatorname{IgG}(r=0.80)$. Lowering renal plasma flow by $24 \%$ during indomethacin therapy in seven patients resulted in a $74 \%$ reduction in proteinuria accompanied by a corresponding diminution of filtrate formed through the shunt pathway. Morphometric analysis of glomerular ultrastructure revealed the magnitude of depression of the glomerular filtration rate and of urinary protein leakage to be related strongly to changes in the epithelial layer of the glomerular capillary wall, but not to the density of subepithelial immune deposits. We conclude $(a)$ that glomerular capillaries in membranous glomerulopathy are characterized by a loss of ultrafiltration capacity and of barrier size-selectivity, and $(b)$ that subepithelial immune deposits do not provide a structural basis for these functional alterations.
\end{abstract}

\section{Introduction}

Membranous glomerulopathy is a common cause of nephrotic range proteinuria in adult males. Although proteinuria may remit spontaneously, it is likely to persist in the majority of instances (1-3). Not infrequently proteinuria is accompanied from the outset by depression of the glomerular filtration rate (GFR), ${ }^{1}$ and a subset of affected patients go on to develop end-stage renal failure within a period of $10 \mathrm{yr}(1-5)$.

Address reprint requests to Dr. Myers, Division of Nephrology, S215, Stanford University Medical Center, Stanford, CA 94305.

Received for publication 16 July 1985

1. Abbreviations used in this paper: $E_{\mathrm{PAH}}$, extraction ratio for para-aminohippuric acid; GFR, glomerular filtration rate; PAH, para-aminohippuric acid.

J. Clin. Invest.

(c) The American Society for Clinical Investigation, Inc.

0021-9738/86/03/0868/10 \$1.00

Volume 77, March 1986, 868-877
Our understanding of the pathogenesis of membranous glomerulopathy derives largely from the availability of an experimental animal model known as Heymann nephritis, which simulates with great accuracy the foregoing clinical features and the pathological characteristics of the human disorder. Active Heymann nephritis is induced by immunizing rodents with an antigen derived from the luminal brush border of tubular epithelial cells (FXIA) (6). Autologous antibodies and complement reach the outer rare layer of the glomerular basement membrane where they form subepithelial immune complexes with a glomerular antigen that has yet to be characterized fully. That the subepithelial immune complexes are formed in situ is suggested by the rapid production of an identical lesion after administration of heterologous antibody to FXIA antigen (passive Heymann nephritis) (7-9). Notwithstanding the accompanying derangement of glomerular capillary wall function, both forms of Heymann nephritis are, like membranous glomerulopathy in humans, characterized by a striking absence of inflammatory or proliferative changes in glomeruli.

In the hope that the functional nature of this injury to the glomerular capillary wall might be elucidated, we have studied 20 adult patients with membranous glomerulopathy and a nephrotic syndrome of recent onset. Determination of the clearance of probe macromolecules (dextrans) of graded size, which are excreted solely by glomerular filtration, was combined with an evaluation of the hemodynamic determinants of glomerular ultrafiltration. We then applied the observed filtration data to a theoretical model of hindered solute transport that accurately represents the human glomerular capillary wall as a heteroporous membrane (10). We also explored the relationship of observed filtration data and derived membrane parameters to glomerular ultrastructure by performing a morphometric analysis of glomeruli obtained by needle kidney biopsy in the same patient population.

\section{Methods}

Patient population. The experimental population was composed of 20 patients with biopsy-proven membranous glomerulopathy who were referred to us consecutively because of nephrotic-range proteinuria $(>4 \mathrm{~g}$ / d). The membranous glomerulopathy was associated with systemic lupus erythematosus in two instances and in one case each with virus B hepatitis and gold therapy of rheumatoid arthritis. In the remaining 16 subjects no known associations of glomerular subepithelial immune complex formation were discernible, and the membranous glomerulopathy was categorized as idiopathic. With two exceptions proteinuria had first been detected in the experimental population between 10 and 36 mo prior to entry into the study. The two exceptions were patients who were found to have proteinuria and membranous glomerulopathy 17 and $7 \mathrm{yr}$ previously. Both experienced prolonged remissions with disappearance of proteinuria. In each case a relapse of the underlying glomerulopathy with reappearance of massive proteinuria became manifest within the 12-mo period preceding the study. 14 patients had received one or more courses of corticosteroid therapy after the diagnosis of membranous glomerulopathy had been established. At the time of study, eight were 
receiving prednisone (range $7.5-90 \mathrm{mg}$ daily), which in two cases was combined with azathioprine $(1.5-2.0 \mathrm{mg} / \mathrm{kg} \cdot \mathrm{d})$. The patient population was predominantly male ( 17 out of 20 ) and ranged in age from 18 to 60 with a mean ( \pm standard error of the mean) of $46 \pm 3$ yr. 20 healthy volunteers spanning the same age range (19-56 yr) served as a control population. This group too was composed mostly of men (12 out of 20). Its members had no history of known renal disease and on examination were found to be normotensive and to have urine that was devoid of measurable protein.

Study protocol. Each patient and volunteer was studied in a clinical research center after giving informed consent to a protocol that had been approved previously by the Committee for the Protection of Human Subjects in Research at Stanford University.

Urine was voided spontaneously after diuresis had been established with oral water loading and, in those with gross edema, with i.v. furosemide administration (80-120 mg). A priming dose of inulin (12.5-25 $\mathrm{mg} / \mathrm{kg}$ ) and para-aminohippuric acid (PAH, $8-16 \mathrm{mg} / \mathrm{kg}$ ) was then administered. Thereafter inulin and PAH were given by continuous infusion to maintain plasma levels constant at $\sim 20$ and $1.5 \mathrm{mg} / \mathrm{dl}$, respectively. With the exception of three patients with a past history of drug allergy, the inulin/PAH prime was followed by a 10 -min i.v. infusion of dextran $40(130 \mathrm{mg} / \mathrm{kg})$.

$60 \mathrm{~min}$ after the priming infusion, arterial blood pressure was determined and blood was sampled for examination of plasma oncotic pressure and plasma protein concentration. Three or four carefully timed urine collections were then made, each of which was bracketed by a blood sample drawn from a peripheral vein. The average inulin clearance for the timed collection periods was taken to represent the GFR. The corresponding value for PAH was equated with renal plasma flow after being divided by an estimated value of the prevailing renal arteriovenous PAH extraction ratio $\left(E_{\mathrm{PAH}}\right)$. In the case of healthy controls, $E_{\mathrm{PAH}}$ was assumed to be 0.85 (11). However, $E_{\mathrm{PAH}}$ has been shown to be moderately depressed in patients with proteinuric glomerular diseases, particularly when GFR is reduced (12-15). To define a representative value of $E_{\mathrm{PAH}}$ in nephrotic subjects with membranous glomerulopathy, we determined this quantity directly on a separate occasion in four members of the experimental group. Renal and peripheral venous blood was sampled simultaneously during a constant infusion of PAH similar to that administered during the clearance study. Although GFR varied widely among these four subjects $\left(41-86 \mathrm{ml} / \mathrm{min}\right.$ per $\left.1.73 \mathrm{~m}^{2}\right), E_{\mathrm{PAH}}$ was distributed over a relatively narrow range measuring $0.58,0.63,0.68$, and 0.73 , respectively. To provide a likely lower bound for renal plasma flow in the present study, we selected arbitrarily a value near the upper end of this range and assumed that $E_{\mathrm{PAH}}$ during the clearance study approximated 0.7 in each member of the experimental population. Fractional macromolecule clearances $\left(\theta_{\mathrm{m}}\right)$ were computed from the first timed collection using the equation:

$\theta_{\mathrm{m}}=(U / P)_{\mathrm{m}} /(U / P)_{\text {in }}$,

where $(U / P)_{m}$ and $(U / P)_{\text {in }}$ refer to the urine-to-midpoint plasma concentration ratio of the macromolecule (dextran, albumin, or $\mathrm{IgG}$ ) and of inulin, respectively.

Upon completion of the baseline clearance study, seven members of the experimental population in whom GFR was $>50 \%$ of the mean control value were selected for further study. Each was given a low sodium diet $(20 \mathrm{meq} / 24 \mathrm{~h})$ and chlorthalidone ( $100 \mathrm{mg}$ daily for $3 \mathrm{~d})$ to induce a state of mild sodium depletion. This was combined with the administration of the cyclooxygenase inhibitor indomethacin, $50 \mathrm{mg} / 8 \mathrm{~h}$ throughout the 3-d period. Commencing $72 \mathrm{~h}$ after the baseline study and within $2 \mathrm{~h}$ of administration of the last dose of indomethacin, the clearance study was repeated using a protocol identical to that of the initial (baseline) study.

Plasma and urinary concentrations of exogenous clearance markers and of endogenous proteins were assayed using techniques that have been described in detail previously (16). The assays for dextran and inulin concentrations were performed after plasma and urine samples from the first clearance period had been submitted to gel permeation chromatog- raphy on precalibrated Sephacryl S300 columns. Plasma oncotic pressure was measured directly using a membrane osmometer (16).

Analysis of glomerular membrane pore structure. Both dextran and inulin are excreted by glomerular filtration and neither secreted nor reabsorbed by the tubule $(17,18)$. Accordingly, the fractional clearance of a dextran molecule of known size (relative to freely filtered inulin) is equal to the Bowman's space-to-plasma water concentration ratio (or glomerular sieving coefficient) for that dextran. To compare the sizeselective properties of the glomerular filter in membranous glomerulopathy to those of normal subjects, we applied the fractional dextran clearances and filtration data to a heteroporous model of the glomerular capillary wall that has been described in detail previously (10). In this model the major portion of the capillary wall is assumed to be perforated by cylindrical pores of identical radius $\left(r_{0}\right)$. The model assumes that there exists, in addition, a parallel "shunt pathway" that does not discriminate on the basis of dextran size, and through which passes a small fraction of filtrate volume. The shunt pathway is characterized by a parameter, $\omega_{0}$, which governs the fraction of filtrate volume $(\langle\omega\rangle)$ passing through this nonselective portion of the membrane. This "isoporous with shunt" model has been shown to provide a more satisfactory representation of fractional dextran clearances in humans than an isoporous model or several other alternative heteroporous models (10). According to this model, the membrane barrier to filtration of water and uncharged macromolecules is fully characterized by the values of $r_{0}, \omega_{0}$, and $K_{f}$; where $K_{\mathrm{f}}$ is the product of effective hydraulic permeability and total glomerular capillary surface area (for two kidneys). The approach used for calculating these intrinsic membrane parameters separates their effects on fractional dextran clearances from those of purely hemodynamic changes.

Morphometric analysis. The biopsies of 15 of the patients with membranous glomerulopathy were performed at our institution. Electron micrographs of these biopsy specimens and of four control specimens of subjects aged 32-62 yr were studied morphometrically. The control specimens were from grossly normal regions of nephrectomy specimens which elsewhere contained a renal cell carcinoma (three cases), or a benign cyst (one case). Specimens were routinely fixed in cacodylate buffered $2 \%$ glutaraldehyde, postfixed in $2 \%$ osmium tetroxide, stained with buffered $1 \%$ uranyl acetate, and embedded in epon. $1-\mu \mathrm{m}$ survey sections were used to locate two or three glomeruli from each specimen, except for three experimental cases in which only a single glomerulus was available for study. $60-90-\mathrm{nm}$ sections were then stained with lead citrate for examination with a transmission electron microscope (Hitachi H300) and photographed at $\times 250, \times 1,000$, and $\times 4,000$. Negatives were enlarged to a uniform print size and a calibration grid $(0.5-\mu \mathrm{m}$ divisions) was similarly photographed to calibrate morphometric measurements. A collage technique was used to encompass entire glomeruli at $\times 1,000$. Components of the filtration barrier were assessed by photographing individual capillary loops at $\times 4,000$.

An SMI Micro-Comp Computerized System (Southern Micro Instruments, Inc., Atlanta, GA) was used to measure distances, count elements, and perform area-perimeter analysis. This system, consisting of a digitizing tablet, computer, and monitor, allows for rapid data acquisition and analysis using the SMI Micro-Comp software package 5.2.2. Area-perimeter analysis was carried out by tracing from an electron micrograph onto the digitizing tablet the perimeter of the structure to be measured. Distances were measured analogously. Measurements included (a) total cross-sectional area of each glomerulus in the sample, (b) fractional mesangial cross-sectional area, $(c)$ total circumferential length of the peripheral capillary loops in each glomerular cross-section, and $(d)$ area and thickness of the capillary wall along the length of individual capillary loops-this was expressed as the ratio of cross-sectional area of all three layers (endothelial, basement membrane, and epithelial) to the length of the capillary loop (Fig. 1), $(e)$ area and thickness of the basement membrane expressed as the ratio of the cross-sectional area of the basement membrane and associated osmophilic immune deposits to the length of the capillary (Fig. 1), $(f)$ the summed area of osmophilic immune deposits per cross-sectional area of the capillary basement 

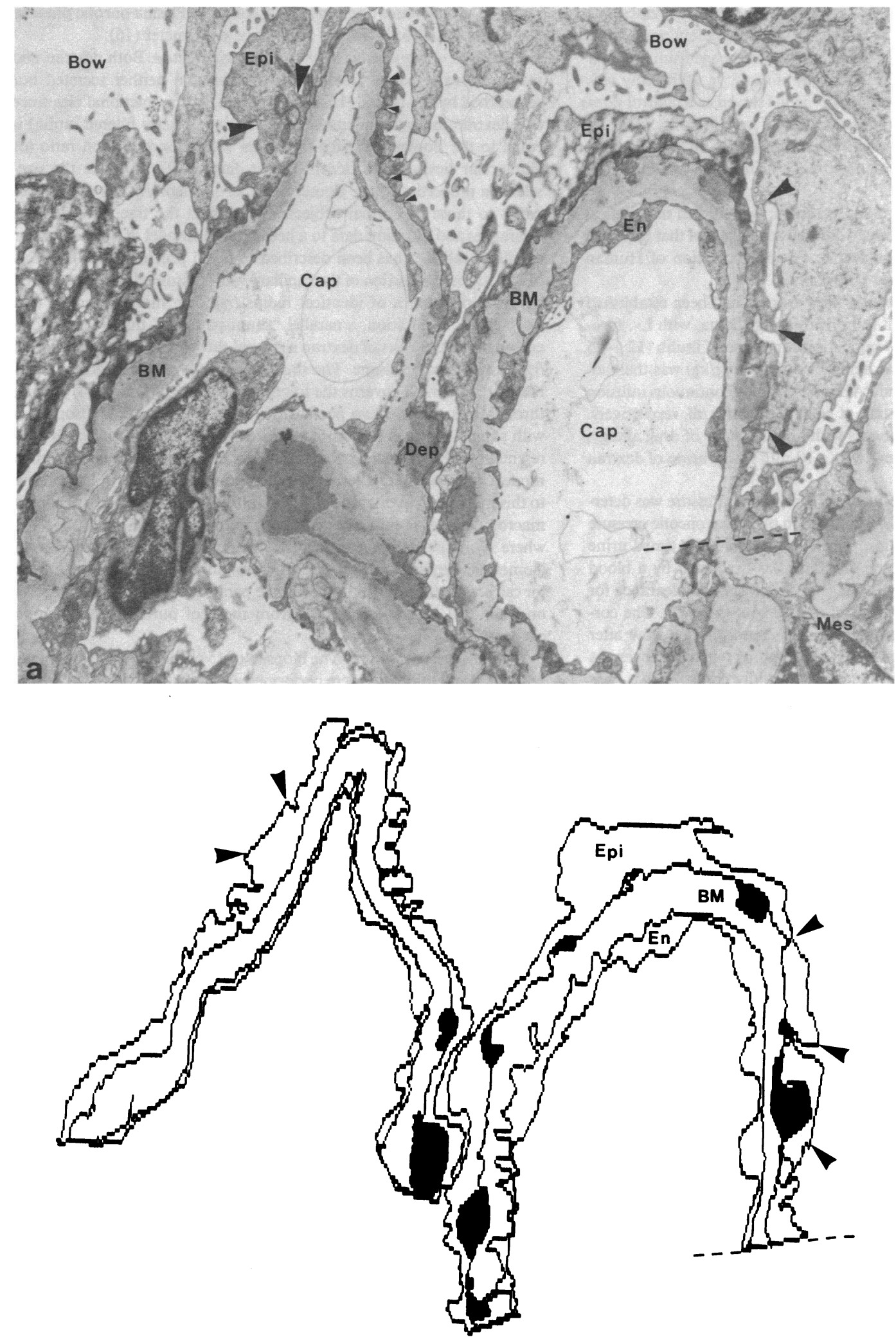

b 
membrane, and $(g)$ the number of epithelial filtration slits per unit length of capillary loop.

The following conventions were used in making the foregoing determinations. The actual outlines of glomerular tufts were traced to determine the cross-sectional area. The criteria of Mauer et al. (19) were used in defining junctions between mesangium and peripheral capillary loops, and loop length was defined as the distance between these junctions. Total thickness of the capillary wall (four above) included epithelial cell foot processes up to the interface with Bowman's space. Connections between foot processes and the main epithelial cell body were included only up to the point at which they were maximally tapered (see Fig. 1). Portions of the main body of epithelial cells were included in measurements of the capillary wall area only where the cells were directly applied to the capillary basement membrane.

Statistical analysis. Differences in clearance and hemodynamic determinations between the membranous glomerulopathy and control populations were evaluated with a two-tailed Student's $t$ test or the Wilcoxon rank sum test for unpaired data. Differences within the membranous glomerulopathy group before and during sodium depletion and indomethacin therapy were evaluated with the Student's $t$ test or the Wilcoxon rank sum test for paired data. The correlations between filtration data and either derived membrane parameters or morphometric measurements of glomerular ultrastructure were evaluated by linear regression analysis. All results are expressed as the means \pm standard error of the mean.

\section{Results}

GFR and determinants. The GFR in patients with membranous glomerulopathy varied over a wide range (15-122 $\mathrm{ml} / \mathrm{min}$ per $1.73 \mathrm{~m}^{2}$, Fig. 2). On the average, however, GFR in the experimental population was depressed to a value only half that of the control group, $58 \pm 7$ vs. $109 \pm 3 \mathrm{ml} / \mathrm{min}$ per $1.73 \mathrm{~m}^{2}(P<0.001$, Table I). By contrast, estimated renal plasma flow was similar in the two groups, $627 \pm 94$ vs. $616 \pm 25 \mathrm{ml} / \mathrm{min}$ per $1.73 \mathrm{~m}^{2}$, respectively. That GFR was selectively or disproportionately lowered relative to renal plasma flow is indicated by a marked lowering of the filtration fraction in patients with membranous glomerulopathy. Whereas the filtration fraction averaged $0.18 \pm 0.01$ in healthy controls, the corresponding value in the experimental population was only $0.12 \pm 0.02(P<0.002)$.

The nephrotic range proteinuria in patients with membranous glomerulopathy was accompanied invariably by hypoproteinemia. As a consequence, measured oncotic pressure of systemic plasma (which is taken to be the same as that prevailing in the afferent arteriole) was severely depressed compared to control values, $15.4 \pm 0.8$ vs. $23.8 \pm 0.5 \mathrm{mmHg}$, respectively $(P$ $<0.001$ ), a finding that should result in an increase in net trans-

Figure 1. (Opposite) (a) A representative micrograph of morphologically mild membranous glomerulopathy shows subepithelial deposits (Dep), open capillary loops (Cap), and no significant increase in basement membrane (BM) thickness. The junction of a mesangial area (Mes) with the capillary wall is indicated by a dashed line. Some filtration slits are indicated by small arrowheads. Filtration slits are reduced in number in comparison to normal glomeruli. Epi, epithelial cell; En, endothelial cell; Bow, Bowman's space. (b) This digitized tracing of the capillary loops from the micrograph shown above demonstrates some of the quantities determined by computer-assisted morphometry. Deposits are indicated in black. As noted in the text, epithelial elements of the wall were only measured when directly applied to the loops. Measurement of the epithelial cell cytoplasm was terminated at the point of maximum tapering as the epithelial processes extended toward the cell bodies (see large arrowheads in the two panels).

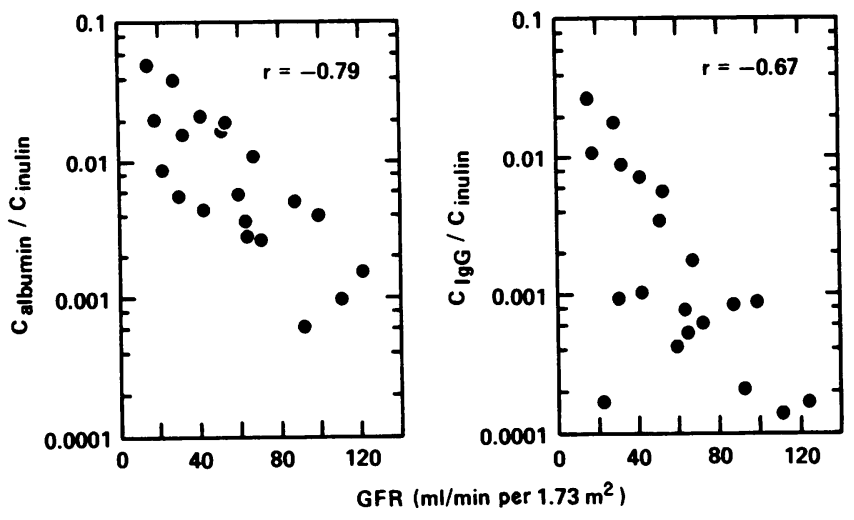

Figure 2. (Left) Fractional albumin clearance plotted as a function of GFR. Coefficient of correlation $=0.79$. (Right) Fractional IgG clearance vs. GFR. Coefficient of correlation $=0.67$. C, clearance.

membrane ultrafiltration pressure. In contrast, mean arterial pressure (estimated as diastolic pressure plus one-third of the pulse pressure) was elevated above the control value of $86 \pm 2$ to $105 \pm 2 \mathrm{mmHg}$ in patients with membranous glomerulopathy $(P$ $<0.001$ ). If glomerular capillary hydraulic pressure was also elevated, as is the case in Heymann nephritis in the rat $(20,21)$, the increase in net transmembrane ultrafiltration pressure in the experimental population would be even greater than inferred from the decline in oncotic pressure alone. This should elevate the GFR and filtration fraction and not lower these quantities as was observed (Table I). By exclusion the glomerular hypofiltration of membranous glomerulopathy seems most likely to result from a profound lowering of the glomerular ultrafiltration coefficient, $K_{\mathbf{f}}$.

Macromolecule excretion and clearance. Neither albumin nor IgG were detectable by radial immunodiffusion in unconcentrated urine of healthy control subjects. In contrast, the urinary excretion of each protein was massively enhanced and easily measurable in all members of the experimental group (Table I). The range of urinary albumin excretion was $1.8-26.3$, averaging $10.0 \pm 1.3 \mathrm{mg} / \mathrm{min}$. The corresponding range of $\mathrm{IgG}$ excreted in

Table I. Renal Function in Membranous Glomerulopathy

\begin{tabular}{lll}
\hline & $\begin{array}{l}\text { Healthy } \\
\text { controls }\end{array}$ & $\begin{array}{l}\text { Membranous } \\
\text { glomerulopathy }\end{array}$ \\
\hline$(n=20)$ & $(n=20)$
\end{tabular}

Glomerular filtration rate

( $\mathrm{ml} / \mathrm{min}$ per $1.73 \mathrm{~m}^{2}$ )

$109 \pm 3$

$58 \pm 7^{*}$

Renal plasma flow

( $\mathrm{ml} / \mathrm{min}$ per $1.73 \mathrm{~m}^{2}$ )

$616 \pm 25$

$627 \pm 94$

Filtration fraction

$0.18 \pm 0.01$

$0.12 \pm 0.02^{*}$

Plasma oncotic pressure

$(\mathrm{mmHg})$

$23.8 \pm 0.5$

$15.4 \pm 0.8^{*}$

Mean arterial pressure

( $m m H g)$

$86 \pm 2$

$105 \pm 2 *$

Urinary albumin excretion ( $m g / m i n)$

NM

$10.0 \pm 1.3$

Urinary IgG excretion

( $m g / m i n)$

NM

$0.7 \pm 0.2$

NM, not measurable.

$* P<0.002$. 
urine was $0.1-2.7$, with an average value of $0.7 \pm 0.2 \mathrm{mg} / \mathrm{min}$. In the face of these massive urinary losses, the serum levels of both albumin $(2.2 \pm 0.2 \mathrm{~g} / \mathrm{dl})$ and $\mathrm{IgG}(0.6 \pm 0.1 \mathrm{~g} / \mathrm{dl})$ were depressed to subnormal values in patients with membranous glomerulopathy.

Assuming minor or negligible fractional protein reabsorption at the high filtered protein loads that characterized the experimental population, the fractional clearances of the test proteins should approach their glomerular sieving coefficients and reflect, with reasonable accuracy, the extent to which the glomerular capillary wall had become hyperpermeable to each protein (22). Accordingly, the relationship of the fractional clearances of albumin and IgG to the GFR in individual patients illustrated in Fig. 2 is instructive. Fractional albumin clearance was correlated inversely with GFR ( $r$ value $=-0.79$ ). An exactly parallel relationship was observed between fractional IgG clearance and GFR $(r=-0.67)$. Both correlations were highly significant, $P$ $<0.001$. Thus the magnitude of glomerular hyperpermeability to both albumin and IgG in membranous glomerulopathy appeared to be associated with the extent to which GFR was depressed.

The fractional clearances of dextrans of broad size distribution differed strikingly between patients with membranous glomerulopathy and control subjects (Fig. 3). The fractional clearances of small, relatively permeant dextrans (radius interval 28-48 A) in membranous glomerulopathy were depressed below normal. The opposite was true of the fractional clearances of large, nearly impermeant dextrans with radii $>50 \AA$, which were elevated above normal. The selective enhancement of fractional clearances of the largest dextrans became magnified with increasing size of the dextran molecule, indicating a glomerular membrane with a less sharp cutoff than normal in patients with membranous glomerulopathy.

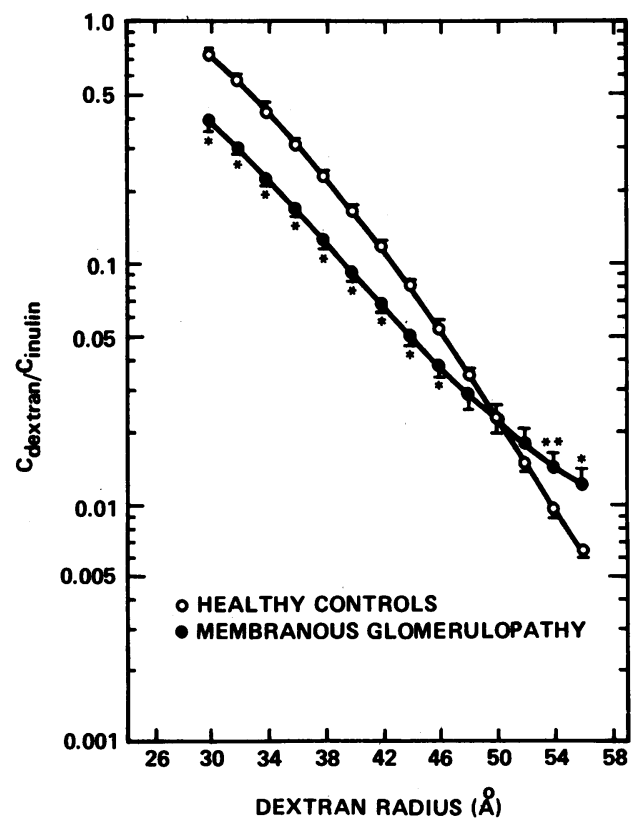

Figure 3. Fractional dextran clearance profile in subjects with membranous glomerulopathy $(\bullet)$ is compared to that of healthy controls (o). Fractional clearances are plotted as a function of effective dextran radius. Results are expressed as mean \pm standard error of the mean; * $P$ $<0.01 ; * * P<0.001$.
Analysis of membrane-pore structure. Values of membrane parameters calculated for the glomerular capillary wall from the mean fractional dextran clearances for each group are shown in Table II. In making these calculations, a value of transmembrane hydraulic pressure difference $(\Delta P)$ similar to that observed in various mammalian species, or $35 \mathrm{mmHg}$, was assumed to be representative of the control population $(23,24)$. In the case of the experimental group, $\Delta \mathrm{P}$ was assumed either to be the same as in normal subjects ( $35 \mathrm{mmHg}$ ) or to be elevated in parallel with arterial pressure to $40 \mathrm{mmHg}$. The latter assumption is more consistent with direct measurements of $\Delta \mathrm{P}$ in glomeruli of rats with Heymann nephritis $(20,21)$.

As shown in Table II, the membrane parameters of the glomerular capillary wall in membranous glomerulopathy differ markedly from those in healthy controls. $K_{\mathrm{f}}$, the glomerular ultrafiltration coefficient, is calculated to be about five times lower, while the radius of the pores that perforate the major portion of the capillary wall $\left(r_{0}\right)$ is smaller by $4 \AA$. In contrast, the membrane parameter that characterizes the shunt pathway, $\omega_{0}$, is about eightfold greater in membranous glomerulopathy than in healthy controls, 0.011 vs. 0.0015 , respectively. The calculated fraction of macromolecule-rich filtrate volume that permeates the shunt pathway $(\langle\omega\rangle)$ is also substantially higher in membranous glomerulopathy than in controls, 0.013 vs. 0.004 , indicating the prominence of the shunt pathway in the experimental population. It is noteworthy that the departure from normality of all three membrane parameters in membranous glomerulopathy is largely independent of whether or not $\Delta P$ is assumed to have become elevated in this disorder (Table II).

When the fractional dextran clearances of each individual control and patient were subjected to analysis in the manner depicted for the mean group data in Table II, remarkably similar results are obtained. Assuming a value for $\Delta \mathrm{P}$ of $35 \mathrm{mmHg}$ in control subjects, $K_{\mathrm{f}}$ averaged $14.7 \pm 1.1 \mathrm{ml} /(\mathrm{min} \cdot \mathrm{mmHg})$, while $r_{0}$ averaged $57.7 \pm 0.2 \AA$. Assuming a value for $\Delta \mathrm{P}$ of $40 \mathrm{mmHg}$, the corresponding values in patients with membranous glomerulopathy were $2.9 \pm 0.5 \mathrm{ml} /(\mathrm{min} \cdot \mathrm{mmHg})$ and $53.6 \pm 0.5 \AA$, respectively. Both differences were highly significant, $P<0.005$. Judged by respective mean values of $\omega_{0}$ of $0.0104 \pm 0.0023$ vs. $0.0015 \pm 0.0002(P<0.001)$, and of $\langle\omega\rangle$ of $0.012 \pm 0.002$ vs. $0.004 \pm 0.001(P<0.001)$, the shunt pathway was significantly more prominent in patients with membranous glomerulopathy than in healthy control subjects. Moreover, when plotted as a function of the corresponding fractional protein clearance (Fig. 4) $\langle\omega\rangle$ was correlated strongly in individual patients with the fractional clearance of both albumin $(r$ value $=0.76, P<0.001)$

Table II. Glomerular Membrane Parameters

\begin{tabular}{llll}
\hline & & \multicolumn{2}{c}{ Membranous glomerulopathy } \\
\cline { 4 - 4 } & $\begin{array}{c}\text { Healthy controls } \\
(\Delta \mathrm{P}=35 \mathrm{mmHg})\end{array}$ & $(\Delta \mathrm{P}=35 \mathrm{mmHg})$ & $(\Delta \mathrm{P}=40 \mathrm{mmHg})$ \\
\hline$K_{\mathrm{f}}(\mathrm{ml} / \mathrm{min}$ & & & \\
$\cdot \mathrm{mmHg})$ & 14.5 & 3.8 & 2.9 \\
$r_{0}(\bar{A})$ & 57.4 & 53.8 & 53.8 \\
$\omega_{0}$ & 0.0015 & 0.0113 & 0.0112 \\
$\langle\omega\rangle$ & 0.0040 & 0.0134 & 0.0130
\end{tabular}

$K_{f}$, ultrafiltration coefficient; $r_{0}$, pore radius of major membrane component; $\omega_{0}$, shunt parameter; $\langle\omega\rangle$, fraction of filtrate volume through shunt. 

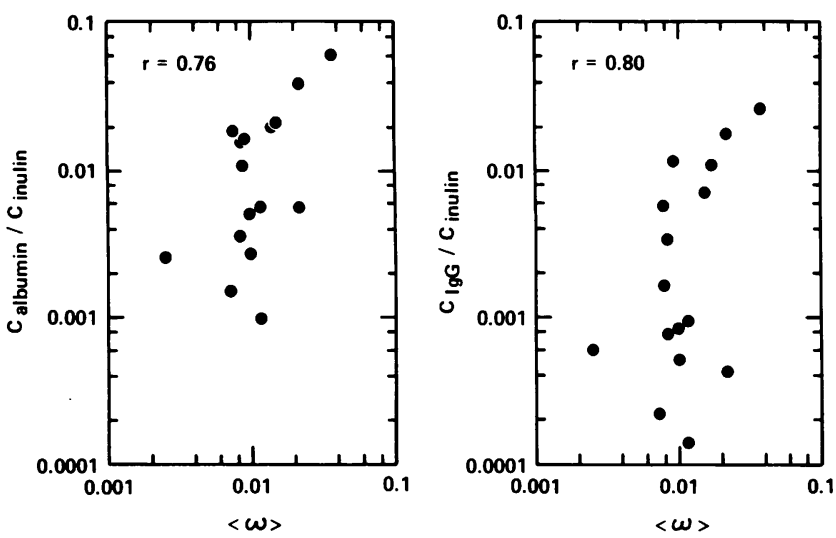

Figure 4. Relationship between fractional protein clearance and the fraction of glomerular filtrate permeating shunt pathway, $\langle\omega\rangle$ in patients with membranous glomerulopathy. (Left) Fractional albumin clearance vs. $\langle\omega\rangle$. (Right) Fractional IgG clearance vs. $\langle\omega\rangle$.

and of $\operatorname{IgG}$ ( $r$ value $=0.80, P<0.001$ ). Thus, as judged by a considerable increase in the participation in the filtration process of a nondiscriminatory shunt pathway $\left(\omega_{0}\right.$ and $\left.\langle\omega\rangle\right)$, there is a loss of membrane size selectivity in membranous glomerulopathy, and the magnitude of urinary protein leakage in this disorder appears to be a function of the fraction of filtrate volume that permeates the shunt pathway. The glomerular membrane in membranous glomerulopathy also differs from normal in that a marked reduction in $K_{\mathrm{f}}$ would appear to account for a substantial increase in transmembrane resistance to water flow, and hence, the observed reduction of GFR.

Correlates of sodium depletion and indomethacin therapy. In keeping with the sodium-depleted state, seven members of the experimental group demonstrated a highly significant weight loss from $80.3 \pm 4.0$ to $77.8 \pm 3.8 \mathrm{~kg}(P<0.01)$ during $3 \mathrm{~d}$ of sodium restriction and diuretic therapy. The simultaneous administration of indomethacin led to a significant reduction by $33 \%$, respectively, of GFR from $76 \pm 10$ to $51 \pm 6 \mathrm{ml} / \mathrm{min}$ per $1.73 \mathrm{~m}^{2}(P<0.005)$. There was a parallel fall by $24 \%$ of renal plasma flow from $812 \pm 95$ to $615 \pm 90 \mathrm{ml} / \mathrm{min}$ per $1.73 \mathrm{~m}^{2}(P$ $<0.05)$. Arterial pressure $(103 \pm 5$ to $96 \pm 5 \mathrm{mmHg}$ ) and plasma oncotic pressure $(15.5 \pm 0.9$ to $21.5 \pm 2.1 \mathrm{mmHg}, P<0.005)$ were restored toward normal, suggesting a likely fall in net glomerular transmembrane ultrafiltration pressure. These hemodynamic changes were accompanied by a remarkable reduction in urinary protein excretion. Albumin excretion fell from an initial value of $10.0 \pm 3.3$ to $2.7 \pm 0.5 \mathrm{mg} / \mathrm{min}$ while urinary IgG excretion declined from $0.7 \pm 0.3$ to $0.1 \pm 0.07 \mathrm{mg} / \mathrm{min}(P<0.02)$ during indomethacin therapy. The corresponding fractional clearance of each protein was reduced by $57 \%$ and $75 \%$, respectively.

Sodium depletion and indomethacin therapy of this subset of the experimental population was associated also with a marked alteration of the fractional dextran clearance profile (Fig. 5). The small radius end of the profile $(<50 \AA)$ was elevated by the hemodynamic perturbation, reaching statistical significance for relatively permeant dextran fractions in the radius interval 36$44 \AA$ A. In contrast, the large radius end of the fractional dextran clearance profile ( $>52 \AA$ ), although also slightly elevated, did not differ significantly from baseline values before indomethacin therapy. The calculated membrane parameters are summarized in Table III. Here it has been assumed that $\Delta \mathbf{P}$ either remained unchanged from baseline at $40 \mathrm{mmHg}$ or, more likely, declined

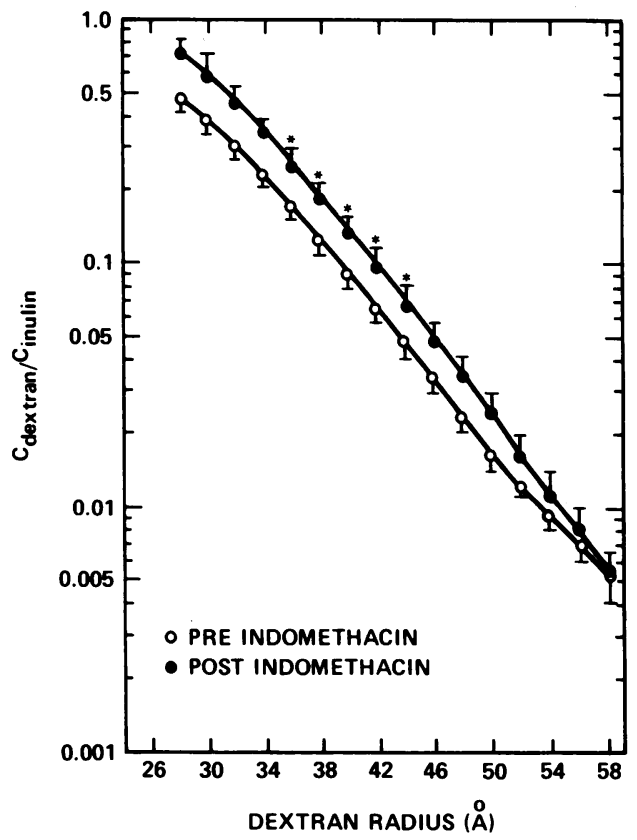

Figure 5. Fractional dextran clearances vs. dextran radius in seven subjects with membranous glomerulopathy before $(0)$ and after $(\bullet)$ indomethacin therapy. ${ }^{*} P<0.05$.

to $35 \mathrm{mmHg}$ in association with the increase in renal vascular resistance and fall in arterial pressure induced by sodium depletion and cyclooxygenase inhibition $(24,25)$. The experimental perturbation had relatively little effect on calculated $K_{\mathrm{f}}$ but restored $r_{0}$ from an initially reduced value of 54.4 toward the control value, $57.4 \AA$. As judged by a reduction in $\omega_{0}$ from 0.0050 to $0.0035-0.0042$, the shunt pathway was reduced in magnitude. From the product of $\langle\omega\rangle$ (the fraction of filtrate volume permeating the shunt pathway) and GFR, we estimate that the absolute rate of formation of macromolecule-rich filtrate through the shunt pathway declined by $40 \%$ from 0.48 to between 0.28 and $0.29 \mathrm{ml} / \mathrm{min}$ per $1.73 \mathrm{~m}^{2}$ (Table III). We interpret these computations to indicate that lowering the glomerular perfusion rate and ultrafiltration pressure restores membrane-pore structure towards normal control values in patients with membranous glomerulopathy. Reduction of proteinuria appears to result, at least in part, from diminution of the shunt pathway in the sizeselective barrier of the filter.

Morphometric analysis. The morphometric parameters of the experimental and control groups are compared in Table IV.

Table III. Effects of Indomethacin on Membrane Parameters

\begin{tabular}{|c|c|c|c|}
\hline & \multirow{2}{*}{$\begin{array}{l}\text { Before } \\
(\Delta P=40 \mathrm{mmHg})\end{array}$} & \multicolumn{2}{|l|}{ After } \\
\hline & & $(\Delta \mathrm{P}=40 \mathrm{mmHg})$ & $(\Delta \mathrm{P}=35 \mathrm{mmHg})$ \\
\hline \multicolumn{4}{|l|}{$K_{\mathrm{f}}(\mathrm{ml} / \mathrm{min}$} \\
\hline$\cdot \mathrm{mmHg})$ & 3.77 & 2.86 & 3.89 \\
\hline$r_{0}(\AA)$ & 54.40 & 57.40 & 57.40 \\
\hline$\omega_{0}$ & 0.0050 & 0.0042 & 0.0035 \\
\hline$\langle\omega\rangle$ & 0.0063 & 0.0055 & 0.0056 \\
\hline $\mathrm{GFR} \cdot\langle\omega\rangle(\mathrm{ml} / \mathrm{min})$ & 0.48 & 0.28 & 0.29 \\
\hline
\end{tabular}

For abbreviations see Table II. 


\begin{tabular}{|c|c|c|c|c|c|c|}
\hline $\begin{array}{l}\text { Membranous } \\
\text { glomerulopathy }\end{array}$ & GA & Mesangial area/GA & $\begin{array}{l}\text { Peripheral } \\
\text { capillary } \\
\text { length/GA }\end{array}$ & Deposit area/GBM area & $\begin{array}{l}\text { GBM + deposit } \\
\text { area/micrometer } \\
\text { loop length }\end{array}$ & $\begin{array}{l}\text { Filtration } \\
\text { slits/micrometer } \\
\text { loop length }\end{array}$ \\
\hline & $m m^{2} \times 1$ & & & & & \\
\hline \multicolumn{7}{|l|}{ Patient } \\
\hline 1 & 35 & 0.28 & 0.07 & 0.27 & 1.33 & .36 \\
\hline 2 & 55 & 0.21 & 0.10 & 0.20 & 1.00 & .24 \\
\hline 3 & 60 & 0.26 & 0.07 & 0.15 & 2.32 & .43 \\
\hline 4 & 40 & 0.17 & 0.12 & 0.28 & 1.17 & .19 \\
\hline 5 & 61 & 0.28 & 0.08 & 0.22 & 1.58 & .39 \\
\hline 6 & 16 & 0.25 & 0.07 & 0.29 & 1.12 & .29 \\
\hline 7 & 78 & 0.25 & 0.10 & 0.10 & 0.80 & .16 \\
\hline 8 & 120 & 0.13 & 0.06 & 0.50 & 1.78 & .44 \\
\hline 9 & 40 & 0.20 & 0.09 & 0.32 & 1.85 & .19 \\
\hline 10 & 13 & 0.06 & 0.10 & 0.26 & 0.97 & .47 \\
\hline 11 & 39 & 0.33 & 0.08 & 0.15 & 1.36 & .59 \\
\hline 12 & 50 & 0.19 & 0.10 & 0.18 & 1.03 & .28 \\
\hline 13 & 27 & 0.22 & 0.10 & 0.14 & 1.23 & .24 \\
\hline 14 & 22 & 0.15 & 0.08 & 0.19 & 1.27 & .21 \\
\hline 15 & 41 & 0.16 & 0.11 & 0.19 & 1.26 & .30 \\
\hline Mean & 46 & 0.21 & 0.09 & 0.23 & 1.34 & .32 \\
\hline SEM & 7 & 0.02 & 0.00 & 0.03 & 0.10 & .03 \\
\hline \multicolumn{7}{|l|}{ Normal controls } \\
\hline Range $(n=4)$ & $29-63$ & $0.19-0.27$ & $0.11-0.15$ & $0-0$ & $0.44-0.73$ & $0.78-1.12$ \\
\hline
\end{tabular}

GA, glomerular area; GBM, glomerular basement membrane.

The cross-sectional areas of $\mathbf{3 6}$ glomeruli from patients with membranous glomerulopathy were similar to those of 10 control glomeruli, 46.4 vs. $48.5 \mathrm{~mm}^{2} \times 10^{-3}(P=\mathrm{NS})$. No significant difference in the fractional mesangial area was evident between the two groups. In contrast, and in accord with qualitative descriptions, there was a distinct thickening of the entire glomerular capillary wall $(2.7 \pm 0.02$ vs. $1.3-1.6$ per micrometer of loop length), and of the basement membrane plus deposit component of this structure, in membranous glomerulopathy (Table IV, column 5). Immune deposits accounted on average for $0.23 \pm 0.03$ of the total area enveloped by basement membrane, ranging from 0.1 to 0.5 in individual biopsy samples. Membranous glomerulopathy also differed from control in that the circumferential length of peripheral capillary loops was reduced when corrected for glomerular cross-sectional area (Table IV, column 3). Furthermore, broadening of foot processes led to a marked reduction in the frequency of filtration slits, $0.32 \pm 0.03$ vs. 0.78 1.12 per micrometer of capillary loop length in controls.

Of the foregoing morphometric parameters, only the latter quantity could be related to GFR in eight patients in whom the needle biopsy and clearance study were contemporareous $(r$ $=0.78$ ). In these eight subjects there was also a significant but inverse correlation between fractional albumin $(r=-0.72)$, or IgG clearances $(r=-0.74)$ and the frequency of filtration slits. Of the remaining quantities in Table IV only one, the circumferential length of peripheral capillary loops per glomerulus, correlated significantly $(P<0.05)$ with fractional protein clearances. The $r$ values versus fractional albumin and IgG clearances were 0.760 and 0.747 , respectively.

\section{Discussion}

The differential solute clearance technique employed in the present study suggests that the glomerular capillary wall in membranous glomerulopathy differs from normal in two major respects. First, a simultaneous and severe depression of GFR, filtration fraction, and fractional clearances of smaller, relatively permeant dextrans (radius < $48 \AA$ ) points to a lowered $K_{f}(26)$. Moreover, although based upon an indirect approach, our findings are consistent with micropuncture studies in Heymann nephritis in the rat and suggest that transcapillary ultrafiltration pressure in the subjects of the present study was enhanced $(20$, 21). It appears, therefore, that the observed glomerular hypofiltration in human membranous glomerulopathy is caused uniquely, by a depression of $K_{\mathrm{f}}(27)$. Second, the development of massive and nonselective proteinuria in the members of the experimental population was accompanied by a selective increase in the filtration of large, nearly impermeant dextrans of radius $>50 \AA$. This finding can be explained by the emergence in the glomerular capillary wall of a second population of pores relatively few in number but of large size (16). Thus, the functional integrity of the glomerular capillary wall in membranous glomerulopathy appears to be compromised by a simultaneous loss of barrier size-selectivity and intrinsic ultrafiltration capacity.

As reviewed recently, glomerular size-selectivity has been represented in many previous studies by idealizing the filtration barrier as an isoporous membrane, one containing cylindrical pores of uniform size (28). That an isoporous model is incompatible with the glomerular capillary wall of membranous glom- 
erulopathy, however, is readily appreciated by comparison of the fractional dextran clearances of experimental subjects of the present study with those of healthy controls. No single population of glomerular pores of uniform size can simultaneously account for the reduced transglomerular transport of smaller dextrans (radius $<50 \AA$ ) but the enhanced transport of larger dextrans (radius > $50 \AA$ ) illustrated for nephrotic subjects with membranous glomerulopathy relative to healthy controls in Fig. 3. We have accordingly used a recently described heteroporous idealization of the glomerular capillary wall (10). This model assumes that the major portion of the glomerular capillary wall functions as an isoporous membrane, while a minor fraction of filtrate volume passes through a second population of pores, relatively few in number but of sufficiently large size that they fail to restrict the passage of dextrans of up to $58 \AA$ radius.

Our computations reveal that the pores in the major isoporous component of the glomerular membrane in membranous glomerulopathy have an effective radius of only 53-54 Å (Table II). Such functional pores are likely to be, if anything, more restrictive in vivo toward globular proteins than to dextrans of comparable size (29). It seems reasonable to infer, therefore, that because of its large molecular radius $(\sim 55 \AA)$ IgG must pass into Bowman's space exclusively through the enlarged pores of the shunt pathway. In keeping with this probability is the strong correlation among patients with membranous glomerulopathy between the fractional clearance of $\mathrm{IgG}$ and the fraction of filtrate volume that permeates the shunt pathway (Fig. 4). ${ }^{2}$ Moreover, careful examination of Fig. 4 reveals that the values of $\langle\omega\rangle$ are equal to or in excess of fractional IgG clearance, suggesting that the shunt can account entirely for the observed levels of the latter quantity. Why the much smaller shunt pathway in the healthy glomerular capillary wall of control subjects is not accompanied by measurable immunoglobulinuria cannot be ascertained from the present data. Conceivably, the large pores of the control shunt pathway may exhibit a greater degree of charge or shape selectivity than in membranous glomerulopathy, thereby permitting the free passage of large uncharged dextrans while severely restricting proteins of equivalent size (29).

That albuminuria in membranous glomerulopathy may, like immunoglobulinuria, also result primarily from loss of barrier size-selectivity is suggested by an equally strong correlation between fractional albumin clearance and the fraction of filtrate volume that permeates the shunt pathway, $\langle\omega\rangle$ (Fig. 4). In contrast to IgG, the subclasses of which are mostly neutral or cationic, albumin is strongly polyanionic in physiologic solution (16). Its molecular radius of $36 \AA$ is small enough that it could, in theory, pass through the major small-pore component of the membrane, should the glomerular capillary wall become depleted of its fixed, negatively charged glycoprotein components. However, that impaired size rather than charge selectivity might represent the major dysfunction of the filtration barrier in membranous glomerulopathy is suggested by analogy with observations in Heymann nephritis. Rennke and his co-workers (30)

2. The contribution of the hypothetical shunt pathway to the fractional clearance of a given macromolecule is closely related to $\langle\omega\rangle$, the fraction of the filtrate volume passing through these nonselective pores. Because the plasma concentrations of the larger macromolecules increase with distance along a glomerular capillary, the fractional clearance contribution of the shunt must slightly exceed $\langle\omega\rangle(10)$. When the filtration fraction for water is relatively small, as in the present study, this distinction is unimportant. observed a normal pattern of barrier charge-selectivity, manifested by restriction of polyanionic macromolecules and enhanced filtration of polycationic macromolecules, in rats with Heymann nephritis. Although not reported in detail, the same study revealed also a selective increase in the filtration of uncharged dextrans of large radius, not unlike that observed in the human subjects of the present study (31).

The current heteroporous model of the glomerular capillary wall also includes mass balance relations that predict variations in solute concentrations and fluxes along the length of the glomerular capillary (10). In this way it becomes possible to isolate the influence of hemodynamic factors on fractional dextran clearances from that owing to the intrinsic selectivity of the glomerular capillary wall (26). The utility of this approach is illustrated by considering the fractional dextran clearances depicted in Fig. 5. Elevation of transglomerular transport of dextrans of broad size distribution during sodium depletion and indomethacin therapy of a subset of the experimental population suggests that the effective pore radius of the glomerular capillary wall had become larger. Indeed, when hemodynamic alterations associated with this regimen are taken into account, the radius of pores in the major component of the membrane $\left(r_{0}\right)$ is calculated to have increased by $3 \AA$. However, the prominence of the shunt pathway (as reflected by $\omega_{0}$ and $\langle\omega\rangle$ ) has diminished slightly, a finding that could contribute to the observed reduction of proteinuria. Also contributing to reduced urinary protein excretion during indomethacin therapy is the simultaneous reduction of GFR (Table III).

The change in membrane-pore structure induced by sodium depletion and indomethacin also demonstrates that the reduction in $K_{\mathrm{f}}$ and loss of size slectivity that attend membranous glomerulopathy can be dissociated (Table III). Indeed, it may be that elevation of glomerular perfusion rate and/or pressure in the basal, unperturbed state distorts the membrane, thereby enlarging the contribution of the shunt pathway. A similar distortion of membrane-pore structure has been induced by maneuvers that increase glomerular capillary hydraulic pressure in the normal rat $(32,33)$. The same is true of colloid volume expansion of human subjects with the nephrotic syndrome (34). The lowering of glomerular perfusion rate and possible decompression of capillaries that appears to have occurred in the wake of indomethacin therapy may be the proximate events leading to improved size selectivity of, and diminished protein traffic across, the glomerular capillary wall in the experimental subjects of the present study.

Using cytochemical techniques, morphologists have identified a local alteration in the composition and ultrastructure of the glomerular basement membrane adjacent to subepithelial immune complexes in experimental (Heymann) membranous glomerulopathy $(35,36)$. Indeed, when the large anionic protein ferritin (radius $61 \AA$ ) has been used as an ultrastructural tracer, it can be seen to traverse the basement membrane in close proximity to the subepithelial immune complexes $(37,38)$. Ultimate access of ferritin and albumin to Bowman's space was found to occur in areas where the overlying epithelial foot processes had become detached from the lamina rara externa of the basement membrane $(37,38)$. Our evaluation of glomerular ultrastructure in the human subjects of the present study reveals that subepithelial immune deposits are copius, occupying between $10 \%$ and $50 \%$ of total glomerular basement membrane area. Although associated foot process broadening with a consequent reduction 
of the number of epithelial filtration slits was a uniform finding and strongly correlated with the magnitude of fractional protein clearance, we were unable to detect separation of the epithelial foot processes from, and denudation of, the underlying basement membrane. It is probable that routine, clinical electron microscopy is too insensitive to permit clear definition of a structural equivalent of the observed loss of functional barrier size-selectivity. The small number of glomeruli available for study in biopsy specimens and the inferior fixation of biopsy tissue compared to the in situ techniques used in the aforementioned animal studies are likely factors contributing to this insensitivity.

Whereas our morphometric analysis of glomerular ultrastructure has not elucidated the nature of impaired membrane size-selectivity, it has provided some interesting insights into the mechanism of glomerular hypofiltration in membranous glomerulopathy. The circumference of ' glomerular capillary loops tended to be smaller and their basement membranes wider than normal (Table IV). A smaller capillary circumference is predicted to provide less surface area across which filtration can take place; a widened basement membrane might lower hydraulic conductivity by lengthening the pathway for transmembrane water flux. Both changes should lower $K_{\mathrm{f}}$. Surprisingly, among patients undergoing a contemporaneous biopsy and clearance study, loop circumference and wall thickness of glomerular capillaries were unrelated to GFR. If anything, wall thickness tended to be related directly to GFR and not inversely as might be expected $(r=0.62$, $P=$ NS). The only observed morphometric correlation that could satisfactorily explain a low $K_{\mathrm{f}}$ in our experimental population was a marked diminution in the number of epithelial filtration slits per unit length of glomerular capillary loop, a nonspecific finding that is common to all proteinuric states. We wish to emphasize, however, that the number of our observations is small, and that further studies will have to be undertaken to confirm the importance and uniqueness of this association.

If the epithelial foot process broadening and the ensuing reduction of the number of filtration slits associated with membranous glomerulopathy is indeed the basis of the observed reduction of GFR, we submit that the glomerular capillary wall dysfunction in this disorder might be related only indirectly to the physical presence of subepithelial immune complexes. The short course of indomethacin given in the present study is unlikely to have had any effect on the presence or density of immune deposits. Thus, the finding during indomethacin therapy of a restoration towards normal of membrane-pore structure accompanied by diminished urinary protein leakage supports a limited role for subepithelial immune deposits in the genesis of proteinuria in this disorder.

It has been shown that Heymann nephritis in the rodent is associated with activation of the terminal complement pathway and the assembly of membrane attack complexes $(39,40)$. Moreover, blockade of late components of the complement pathway in Heymann nephritis prevents proteinuria, notwithstanding the presence of subepithelial immune complexes $(41$, 42). This phenomenon may explain the apparent lack of association between glomerular capillary wall dysfunction and density of immune deposits in the experimental subjects of the present study. Activation of the terminal complement pathway and the formation of membrane attack complexes has been demonstrated also in human membranous glomerulopathy (43). It may be that complement activation leads to altered perfusion characteristics of the glomerular microcirculation in this circumstance. We propose that such local hemodynamic alterations alone, or in combination with yet other biophysical phenomena, may provide a functional basis for the impaired barrier sizeselectivity and loss of ultrafiltration capacity that typify the glomerular capillary walls of subjects with membranous glomerulopathy.

\section{Acknowledgments}

We acknowledge with gratitude the expert secretarial help of Mary Peterson in the preparation of this manuscript.

This study was supported by grants AM-29985 and AM-20368 from the National Institutes of Health. Dr. Shemesh is a recipient of the John P. Merrill Research Fellowship of the National Kidney Foundation.

\section{References}

1. Row, P. G., J. S. Cameron, D. R. Evans, R. H. R. White, C. S. Ogg, C. Chatler, and C. B. Brown. 1975. Membranous nephropathy. Long term follow-up and association with neoplasia. $Q$. J. Med. 44:207239.

2. Erwin, D. T., J. V. Donadio, and K. E. Holley. 1973. The clinical course of idiopathic membranous nephropathy. Mayo Clin. Proc. 48: 697-712.

3. Noel, L. H., M. Zanetti, D. Droz, and C. Barbanel. 1979. Long term prognosis of idiopathic membranous glomerulonephritis. Study of 116 untreated patients. Am. J. Med. 66:82-89.

4. Franklin, W. A., R. B. Jennings, and D. P. Earle. 1973. Membranous glomerulonephritis. Long term serial observations on clinical course and morphology. Kidney Int. 4:36-45.

5. Collaborative Study of the Adult Idiopathic Nephrotic Syndrome. 1979. A controlled study of short-term prednisone treatment in adults with membranous nephropathy. N. Engl. J. Med. 301:1301-1306.

6. Heymann, W., D. B. Hackel, S. Harwood, J. G. F. Wilson, and J. L. P. Hunter. 1959. Production of nephrotic syndrome in rats by Freund's adjuvant and rat kidney suspension. Proc. Soc. Exp. Biol. Med. 100:660-667.

7. Fleuren, G., J. Grond, and P. J. Hoedemaeker. 1980. In situ formation of subepithelial glomerular immune complexes in passive serum sickness. Kidney Int. 17:631-637.

8. Couser, W. G., D. R. Steinmuller, M. M. Stilmant, D. J. Salant, and L. M. Lowenstein. 1978. Experimental glomerulonephritis in the isolated perfused rat kidney. J. Clin. Invest. 62:1275-1287.

9. Van Damme, B. J. D., G. J. Fleuren, W. W. Bakker, R. L. Vernier, and J. Hoedemaeker. 1978. Experimental glomerulonephritis in the rat induced by antibodies directed against tubular antigens. V. Fixed glomerular antigens in the pathogenesis of heterologous immune complex glomerulonephritis. Lab. Invest. 38:502-510.

10. Deen, W. M., C. R. Bridges, B. M. Brenner, and B. D. Myers. 1985. Heteroporous model of glomerular size-selectivity:application to normal nephrotic humans. Am. J. Physiol. 249:F374-F389.

11. Aurell, M. 1969. Renal response in man to plasma volume expansion and angiotensin. Scand. J. Clin. Lab. Invest. 24:3-59.

12. Bradley, S. E., G. P. Bradley, C. J. Tyson, J. J. Curry, and W. D. Glake. 1950. Renal function in renal disease. Am. J. Med. 9:766798.

13. Brodwall, E. K. 1960. Renal extraction in PAH in renal disease. Scand. J. Clin. Lab. Invest. 16:12-20.

14. Bergstrom, J., H. Bucht, B. Josephson, H. Sundell, and L. Werko. 1960. The renal extraction of para-aminohippurate in normal persons and in patients with diseased kidneys. Scand. J. Clin. Lab. Invest. 11: 361-375.

15. Carghill, W. H. 1948. The measurement of glomerular and tubular plasma flow in the normal and diseased human kidney. J. Clin. Invest. 28:189-192.

16. Myers, B. D., T. B. Okarma, S. Friedman, C. Bridges, J. Ross, S. Asseff, and W. M. Deen. 1982. Mechanisms of proteinuria in human glomerulonephritis. J. Clin. Invest. 70:732-743. 
17. Chang, R. L. S., I. F. Ueki, J. L. Troy, W. M. Deen, C. R. Robertson, and B. M. Brenner. 1975. Permselectivity of the glomerular capillary wall to macromolecules. II. Experimental studies in rats using neutral dextran. Biophys. J. 15:887-895.

18. Chang, R. L. S., W. M. Deen, C. R. Robertson, C. M. Bennett, R. J. Glassock, and B. M. Brenner. 1975. Permselectivity of the glomerular capillary wall. Studies of experimental glomerulonephritis in the rat using neutral dextran. J. Clin. Invest. 57:1272-1280.

19. Mauer, S. M., M. W. Steffes, E. N. Ellis, D. E. R. Sutherland, D. M. Brown, and F. C. Goetz. 1984. Structural-functional relationships in diabetic nephropathy. J. Clin. Invest. 74:1143-1155.

20. Allison, M. E. M., C. B. Wilson, and C. W. Gottschalk. 1974. Pathophysiology of experimental glomerulonephritis in rats. J. Clin. Invest. 53:1402-1423.

21. Ichikawa, I., J. R. Hoyer, M. W. Seiler, and B. M. Brenner. 1982. Mechanisms of glomerulotubular balance in the setting of heterogenous glomerular injury. J. Clin. Invest. 69:185-198.

22. Oken, D. E., B. B. Kirschbaum, and D. M. Landwehr. 1981. Micropuncture studies of the mechanisms of normal and pathologic albuminuria. Contrib. Nephrol. 24:1-7.

23. Ichikawa, I., D. A. Maddox, M. G. Cogan, and B. M. Brenner. 1978. Dynamics of glomerular ultrafiltration in euvolemic Munich-Wistar rats. Renal Physiol. 1:121-131.

24. Navar, L. G., P. D. Bell, R. W. White, R. L. Watts, and R. H. Williams. 1977. Evaluation of the single nephron glomerular filtration coefficient in the dog. Kidney Int. 12:137-149.

25. Schor, N., I. Ichikawa, and B. M. Brenner. 1981. Mechanisms of action of various hormones and vasoactive substances on glomerular ultrafiltration in the rat. Kidney Int. 20:442-451.

26. Chang, R. L. S., I. F. Ueki, J. L. Troy, W. M. Deen, C. R. Robertson, and B. M. Brenner. 1975. Permselectivity of the glomerular capillary wall to macromolecules. I. Theoretical considerations. Biophys. J. 15:887-895.

27. Deen, W. M., C. R. Robertson, and B. M. Brenner. 1972. A model of glomerular ultrafiltration in the rat. Am. J. Physiol. 223:11781183.

28. Deen, W. M., C. R. Bridges, and B. M. Brenner. 1983. Biophysical basis of glomerular permselectivity. J. Membr. Biol. 71:1-10.

29. Rennke, H. G., and M. A. Venkatachalam. 1979. Glomerular permeability of macromolecules: effect of molecular configuration on the fractional clearance of uncharged dextran and neutral horseradish peroxidase in the rat. J. Clin. Invest. 63:713-717.

30. Rennke, H. G., W. G. Couser, Y. Patel, and M. A. Venkatachalam.
1978. Membranous nephropathy: fractional clearance of anionic, neutral and cationic horseradish peroxidase in Lewis rats with autologous immune complex nephritis. Abstracts of the Seventh International Congress of Nephrologists, Montreal. $\mathrm{x}-2$.

31. Arnaout, M. A., H. G. Rennke, and R. S. Cotran. 1981. Membranous glomerulonephritis. In Contemporary Issues in Nephrology. Nephrotic Syndrome. Vol. 9. B. M. Brenner and J. H. Stein, editors. Churchill Livingstone, New York. 199-235.

32. Pessina, A. C., B. Hulme, and W. S. Pert. 1972. Renin-induced proteinuria and the effects of adrenalectomy. II. Morphology in relation to function. Proc. R. Soc. Lond. B Biol. Sci. 180:61-71.

33. Kon, V., and I. Ichikawa. 1983. Role of angiotensin II in altered transglomerular passage of water and albumin induced by renal vein constriction. Am. Soc. Nephrol. Abstr. 16:195A.

34. Friedman, S., C. R. Bridges, W. M. Deen, B. M. Brenner, and B. D. Myers. 1982. Filtration dynamics and barrier dysfunction in glomerulonephritis. Am. Soc. Nephrol. Abstr. 15:121A.

35. Kelley, V. E., and T. Cavallo. 1977. Glomerular permeability. Ultrastructural studies in New Zealand black/white mice using polyanionic ferritin as a molecular probe. Lab. Invest. 37:265-274.

36. Schneeberger, E. E., G. Stavrakis, and K. McCarthy. 1983. Alterations in glomerular anionic sites in autologous immune complex nephritis. Lab. Invest. 49:445-452.

37. Schneeberger, E. E., and W. E. Grupe. 1976. The ultrastructure of the glomerular slit diaphragm in autologous immune complex nephritis. Lab. Invest. 34:298-308.

38. Schneeberger, E. E., M. A. O'Brien, and W. E. Grupe. 1979. Altered glomerular permeability in Munich-Wistar rats with autologous immune complex nephritis. Lab. Invest. 40:227-236.

39. Salant, D. J., S. Belok, M. P. Madaio, and W. G. Couser. 1980. A new role for complement in experimental membranous nephropathy in rats. J. Clin. Invest. 66:1339-1350.

40. De Heer, E., M. R. Daha, S. Bhakdi, H. Bazin, and A. Van Es. 1985. Possible involvement of terminal complement complex in active Heymann nephritis. Kidney Int. 27:388-393.

41. Adler, S., P. J. Baker, P. M. Pritzl, and W. G. Couser. 1984. Presence of terminal complement components in complement-dependent experimental glomerulonephritis. Kidney Int. 26:397-403.

42. Baker, P. J., R. F. Ochi, S. Adler, R. J. Johnson, and W. G. Couser. 1985. C $_{6}$ depletion abolishes proteinuria in experimental membranous nephropathy. Clin. Res. 33:475A. (Abstr.)

43. Falk, R. J., A. P. Dalmasso, Y. Kim, C. H. Tsai, J. I. Scheinman, H. Gewurz, and A. F. Michael. 1983. Neoantigen of the polymerized ninth component of complement. J. Clin. Invest. 72:560-573. 